\title{
Cycle de l'azote dans les sédiments des lacs de gravière : étude d'un cas par traçage isotopique en microcosme
}

\author{
L. Labroue ${ }^{1}$ \\ C. Helmer de Almeida ${ }^{1}$
}

Mots clés : Azote, sédiments, ${ }^{15} \mathrm{~N}$, lacs de gravières, bioturbation.

Des sédiments d'un lac de gravière mésotrophe ont été mis à incuber au laboratoire pendant 15 jours en présence ou en l'absence de vers oligochètes. L'apport d'azote ammoniacal marqué par l'isotope lourd $\left({ }^{15} \mathrm{~N}\right)$ a permis de mesurer les flux entre les différents compartiments azotés ( $\mathrm{N}$ organique, $\mathrm{N}-\mathrm{NH}_{4}{ }^{+}, \mathrm{N}-\mathrm{NO}_{3}{ }^{-}$). La nitrification apparaît négligeable alors que la dénitrification, un peu plus de $1 \mathrm{mg} \cdot \mathrm{m}^{-2} \mathrm{~h}^{-1}$, est alimentée par les nitrates allochtones de la nappe. La perte d'azote ammoniacal par volatilisation est plus importante que la dénitrification mais elle est probablement influencée par le système d'aération. L'influence des oligochètes n'apparaît sensible qu'au niveau du couple minéralisation - réorganisation qu'elle active nettement.

\section{Nitrogen cycling in the sediments of gravel-pit lakes : a case study by isotopic tracer in a microcosm}

Keywords : Nitrogen, sediment, ${ }^{15} \mathrm{~N}$, gravel-pit lakes, bioturbation.

Sediments from mesotrophic gravel-pit lakes with and without oligochaete worms were incubated in the laboratory during 15 days. ${ }^{15} \mathrm{~N}-\mathrm{NH}_{4}{ }^{+}$was added in order to measure rates of fluxes between organic $\mathrm{N}, \mathrm{NH}^{+}$and $\mathrm{NO}^{-}$pools. Nitrification appeared negligible while denitrification, a little more than $1 \mathrm{mg} \cdot \mathrm{m}^{-2} \mathrm{~h}^{-1}$, is fed by allochtonous ground-water NO3- The loss of ammoniac nitrogen by volatilization was important but likely influenced by the aeration design. The influence of the oligochaete worms was only appreciable at the level of the mineralization - assimilation pair whose rates were markedly enhanced.

Le rôle épurateur des lacs de gravières vis-à-vis de la pollution nitratée des nappes alluviales qui les traversent a été démontré et quantifié dans la vallée de la Garonne (France) au cours des années 1980 (Bergé 1983, Labroue et al., 1988). Cependant, le rôle des sédiments dans ce processus est connu depuis longtemps (Keeney et al., 1971). Le cycle interne microbien de l'azote dans les sédiments peut être représenté de la façon suivante (Fig.1).

\section{Introduction}

Comme on le voit, il existe des flux antagonistes simultanés dont la valeur brute n'est pas accessible par la simple analyse chimique. Par exemple, l'absence de

1. Centre d'Ecologie des Systèmes Aquatiques Continentaux UMR C 5576 CNRS/Université Paul Sabatier, 118 route de Narbonne, F - 31062 Toulouse Cedex 4. nitrate ne signifie pas l'absence de nitrification puisqu'il peut il y avoir dénitrification simultanément. Seul, le traçage isotopique donne accès à cette connaissance. Dans la compréhension du rôle épurateur des lacs de gravière, il était important de savoir quelles étaient les parts respectives de la dénitrification liée aux apports allochtones (nappe) et de celle liée à la nitrification dans les sédiments (couplage nitrificationdénitrification). D'autre part, les paramètres de l'activité microbienne sont influençés par les organismes benthiques tels les Chironomidés et les Oligochètes. Après avoir étudié l'influence des Tubificidés sur le cycle du phosphore dans ces lacs (Guérin \& Labroue 1991), il était naturel de s'interroger sur leur rôle dans le cycle de l'azote. A cet effet, nous avons mis à incuber en microcosmes, avec ou sans Oligochètes, des sédiments prélevés dans un lac de gravière mésotrophe représentatif des innombrables ballastières qui mitent la basse plaine de la Garonne aux alentours de Toulouse. 


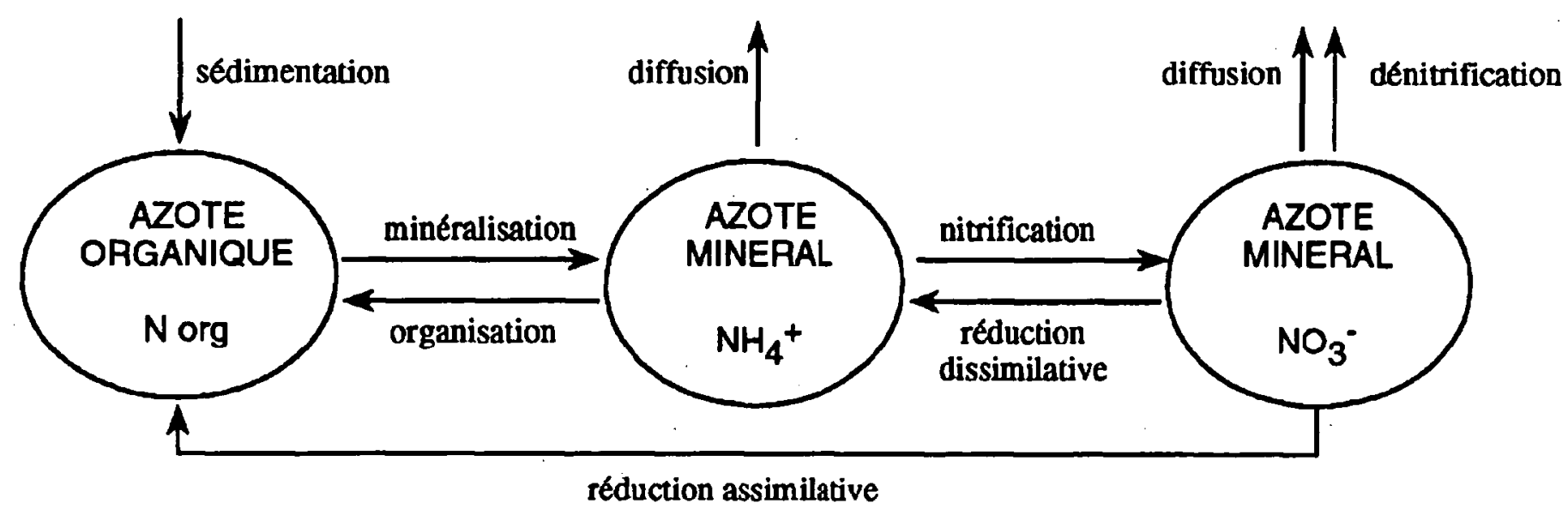

Fig. 1. Cycle de l'azote dans les sédiments à l'échelle microbienne.

Fig. 1. Nitrogen transformation in sediment at the microbial scale.

L'utilisation de l'isotope lourd ${ }^{15} \mathrm{~N}$ dans la mise en évidence des processus biologiques date des années 1940. Des modèles de fonctionnement du cycle interne de l'azote dans les sols ont été établis dans les années 1950 (Kirkham \& Bartholomew 1954, 1955). Dans la colonne d'eau des lacs et des océans, les études se sont développées dans les années 1960 (Goering \& Dugdale 1966, Dugdale \& Goering 1967). Dans les sédiments, marins essentiellement, les investigations sont plus tardives (Goering \& Pamatmat 1970, Chen et al. 1972, Tiren et al. 1976, Sørensen 1978, Koike \& Hatori 1978, Madsen 1979, Oren \& Blackburn 1979, Blackburn 1979 et d'autres). En 1983, Blackburn et Henricksen modélisent entièrement le cycle de l'azote dans les sédiments marins d'une station du Kattegat (Danemark).

Le rôle de la bioturbation dans le cycle de l'azote a déjà été mis en évidence par Kikuchi \& Kurihara (1982) dans les rizières, par Chaterpaul et al.(1979) dans les rivières et par Gardner et al. (1983) dans les sédiments des lacs. Cependant les études de ce type dans les milieux d'eau douce restent peu nombreuses, en raison sans doute de la lourdeur et du coût du traçage isotopique de l'azote.

\section{Matériel et méthodes}

\section{1. Protocole expérimental}

Les sédiments nécessaires à l'expérience ont été collectés dans le lac de gravière dit des «Bedels», situé dans la commune de Saint Jory, à une vịngtaine de kilomètres au nord de Toulouse (France). Il s'agit d'un lac mésotrophe (teneur moyenne en chlorophylle a de $11 \mathrm{mg}^{-\mathrm{l}^{-1}}$ ), de $2,5 \mathrm{~m}$ de profondeur moyenne. Les paramètres principaux des sédiments sont donnés par le tableau 1. Ces sédiments ont été recueillis grâce à une drague du type de celle d'Eckman. Ce matériel, transporté au laboratoire, a été tamisé afin de récupérer les Oligochètes et autres animaux benthiques. Le sédiment a ensuite été entreposé à l'obscurité en chambre froide en attendant le début de l'expérience (environ 1 semaine).

Pour réaliser l'étude, deux aquariums du commerce $(21 / 38 / 25 \mathrm{~cm})$ en plexiglass ont été utilisés avec quelques modifications afin de permettre une agitation et une aération théoriquement étanche (cf Fig. 2) :

- l'un, désigné aquarium $\mathrm{A}$, devant recevoir du sédiment et des annélides oligochètes Tubifex tubifex Müll. (900 individus environ soit $11000 / \mathrm{m}^{2}$ ce qui représente une valeur moyenne pour ce type de lac).

- l'autre, aquarium $S$, ne contenant que du sédiment dépourvu d'Oligochètes.

Tableau 1. Paramètres principaux des sédiments du lac exprimés en \% de volume (1) ou de matière sèche (2).

Table 1. Main parameters of the lake sediment expressed as volume percent (1) or dry matter mass percent (2).

\begin{tabular}{ccccc}
\hline Porosité (1) & Carbone (2) & Azote (2) & Phosphore (2) & Carbonates (2) \\
$64 \pm 16$ & $2,2 \pm 1,4$ & $0,18 \pm 0,06$ & $0,07 \pm 0,01$ & $24,7 \pm 6,0$ \\
\hline
\end{tabular}




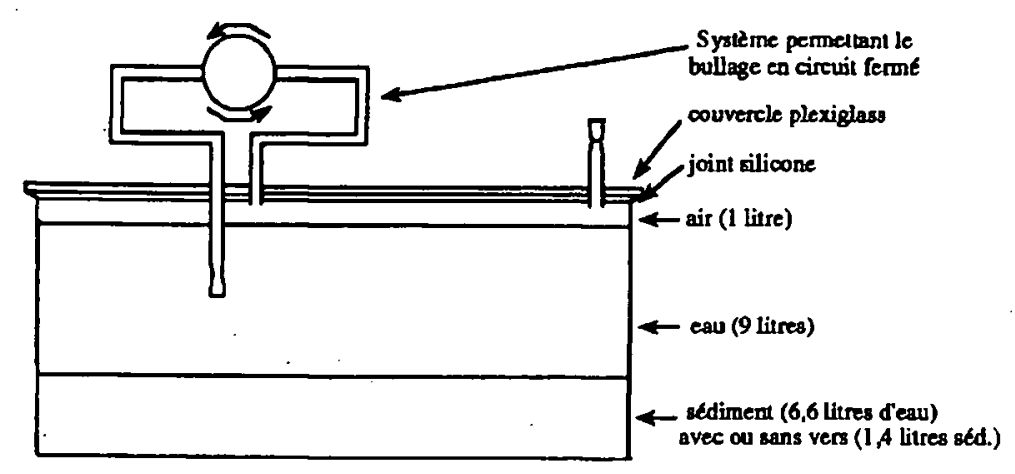

Fig. 2. Dispositif expérimental d'incubation des sédiments en conditions aérées et agitées dans un récipient étanche.

Fig. 2.Experimental design developed to incubate sediment in agitated and aerated conditions in a closed reservoir.

En début d'expérience $(t=0)$, nous avons introduit dans chacun d'eux 9 litres d'eau contenant $20,9 \mathrm{mg}$ de ${ }^{15} \mathrm{~N}-\mathrm{NH} 44^{+}$marqué à $98 \%$, soit environ $15 \%$ de l'azote ammoniacal présent naturellement. Nous avons ajouté ensuite à ces 9 litres d'eau, un mélange sédiment-eau jusqu'à atteindre une hauteur totale (eau + sédiment) de $22 \mathrm{~cm}$. Les conditions sont sensées représenter la phase de repos suivant une remise en suspension. La remise en suspension des sédiments est un phénomène fréquent dans ce type de gravière peu profonde où plus de $90 \%$ des sédiments recueuillis dans des pièges tubulaires proviennent de leur remise en suspension. L'étanchéité de chacun des aquariums a été ensuite réalisée grâce à la mise en place d'un joint silicone entre le couvercle et le bac (Fig. 2) mais le procédé s'est avéré inefficace. Un système de bullage par pompe péristaltique en circuit fermé a été installé afin d'assurer l'agitation modérée et l'oxygénation de l'eau surnageante. Il n'a été mis en route que 24 heures plus tard, afin de laisser au sédiment le temps de se déposer. Les deux aquariums sont ensuite laissés à l'obscurité à une température de $22^{\circ} \mathrm{C}$.

Deux semaines plus tard ( $\mathrm{t}=352$ heures), l'expérience a été arrêtée. Des prélèvements d'air ont été réalisés avant l'ouverture et, une fois les aquariums ouverts, des carottes de sédiment et des échantillons d'eau ont été recueillis pour les analyses chimiques, effectuées en double ou triple exemplaire. Le dosage de l'azote ammoniacal a été effectué par colorimètrie au bleu d'indophénol, l'ammonium adsorbé étant extrait par une solution de $\mathrm{KCl}$ normale. Le dosage des nitrates et des nitrites (négligeables) a été réalisé sur une chaîne d'auto-analyseur Technicon, les nitrates étant réduits sur une colonne cadmium-cuivre. L'azote organique a été minéralisé selon Kjeldahl, après destruction des nitrates et extraction de l'ammonium déjà présent. Le dosage et la récupération de l'ammonium formé a été effectué par distillation dans l'acide sulfurique dilué, pour être utilisé en analyse isotopique (Bremner 1965).

Chaque compartiment (eau superficielle, eau inter-. stitielle et phase solide) a été entièrement récupéré pour les déterminations d'excès isotopique pour chaque espèce chimique $\left(\mathrm{NH}^{+}, \mathrm{NO}^{-}, \mathrm{N}\right.$ organique). Les analyses isotopiques ont été réalisées en triple exemplaire. L'azote diatomique a été analysé sur un échantillon gazeux de la phase atmosphérique. La teneur en solution, déduite de la loi de Henry, est négligeable.

\section{2. Analyses de l'azote 15}

Les différentes formes d'azote ont été transformées en azote ammoniacal selon les méthodes préconisées par Bremner (1965). L'azote ammoniacal est ensuite oxydé en azote diatomique gazeux par l'hypobromite de lithium sous vide (Mariotti et al. 1978). L'abondance isotopique des échantillons est déterminée sur un spectromètre de masse T.H.N.204 pour les excès isotopiques supérieurs à $0,1 \%$ et par un spectromètre V.G.602 à double collection et double introduction pour les valeurs inférieures (N-NO3- et air) en utilisant les trois pics de fréquences : la masse 28 correspondant à la molécule ${ }^{14} \mathrm{~N}^{14} \mathrm{~N}$, la masse 29 correspondant à la molécule $14 \mathrm{~N} 15 \mathrm{~N}$ et la masse 30 à la molécule ${ }^{15} \mathrm{~N}^{15} \mathrm{~N}$.

Rappelons que l'abondance isotopique (\%) est définie par :

nombre d'atomes 15

$$
A=\frac{}{\text { nombre d'atomes } 14+\text { nombre d'atomes } 15}
$$

L'excès isotopique ( $\mathrm{E} \%)$ est la différence entre l'abondance isotopique de l'échantillon et l'abondance naturelle atmosphérique soit $0,3663 \%$.

\section{3. Exploitation des résultats}

Les traceurs permettent de mettre en évidence une transformation précurseur-produit et de mesurer sa vitesse, dans certaines conditions, souvent supposées. Par exemple, les compartiments doivent être marqués de manière uniforme en ce qui concerne les divers sites réactionnels. Le traceur doit montrer des compartiments physiques, chimiques et biologiques identiques à ceux de l'isotope commun, ce qui n'est pas toujours le cas : il peut y avoir fractionnement isotopique, phénomène utilisé lors du traçage isotopique naturel. Plus généralement, on ajoute une certaine quantité de traceur (träçage isotopique artificiel), mais à des doses suffisamment faibles pour ne pas trop perturber le système. Cet impératif est quelque peu contradictoire 
avec les exigences de la détection. L'addition de traceur peut se faire en dose unique (technique de chasse) ou en continu (technique de charge). La première méthode est la plus employée.

La concentration en traceur du compartiment marqué évolue au cours du temps du fait de ses échanges avec les compartiments voisins. Lorsque ces compartiments sont bien connus et lorsque la cinétique peut être décrite par des équations exponentielles, on peut procéder à une analyse compartimentale selon un modèle déterministe (Shipley \& Clark 1972). Cette analyse est riche en information mais pratiquement inutilisable en milieu naturel (Fardeau \& Jappe 1982, Labroue et al. 1985). On considère alors le compartiment comme une «boîte noire» dans lequel existe un temps moyen de transit du groupe étudié: c'est le «modèle stochastique». Un calcul rigoureux du flux à la sortie d'un compartiment peut être effectué si la cinétique de la concentration en traceur peut être suivie jusqu'à épuisement de la dose injectée (équation de Stewart-Hamilton); mais la durée des expériences en laboratoire est généralement trop courte, ceci afin d'éviter une modification trop importante des conditions initiales.

Même sur de courtes périodes, les compartiments et les flux sont rarement suffisamment stables pour que l'on puisse éviter de tenir compte de leur évolution. Une solution approchée passe par la résolution conjointe de deux équations (Shipley \& Clark 1972) :

- l'une décrivant l'évolution du traceur dans le compartiment marqué

$$
\mathrm{dq} / \mathrm{dt}=-\mathrm{F} 2 . \mathrm{E}(\mathrm{t})
$$

avec $\mathrm{q}=$ quantité de traceur dans le compartiment, $F 2=$ flux de sortie et $E(t)=$ excès isotopique du traceur au temps $\mathrm{t}$;

- l'autre décrivant la variation de l'élément commun dans ce même compartiment

$$
\mathrm{dQ} / \mathrm{dt}=\mathrm{F} 1-\mathrm{F} 2
$$

avec F1 flux d'entrée dans le compartiment, F2 flux de sortie.

Si les flux peuvent être considérés comme constants, on peut utiliser l'équation suivante (Shipley \& Clark 1972) qui suppose une décroissance exponentielle du traceur :

$$
\mathrm{F} 1=-(\mathrm{Q} 1-\mathrm{Q} 2) \ln (\mathrm{E} 1-\mathrm{E} 2)
$$$$
(\mathrm{t} 2-\mathrm{t} 1) \ln (\mathrm{Q} 1 / \mathrm{Q} 2)
$$

En présence d'un système non stationnaire et à flux variable, ce qui est généralement le cas dans les expériences en microcosmes, on peut admettre une évolution linéaire sur de courtes périodes :

$$
\mathrm{F} 2=\frac{[(\mathrm{Q} 1+\mathrm{Q} 2) / 2] \cdot(\mathrm{E} 2-\mathrm{E} 1)}{(\mathrm{t} 2-\mathrm{t} 1) \cdot(\mathrm{E} 1+\mathrm{E} 2) / 2}
$$

Le modèle exponentiel a été adopté par Caperone (1979), Blackburn (1979), Glibert et al. (1982) et Sumi \& Koike (1990) pour le compartiment ammoniacal.

Le modèle linéaire a été utilisé par Guiraud (1984) et par Sumi \& Koike (1990) pour le compartiment nitrate.

Les compartiments non directement marqués reçoivent le traceur du compartiment marqué initialement puis le transmettent à d'autres. Dans leur cas, l'équation.(1) devient :

$$
\mathrm{dq} / \mathrm{dt}=\mathrm{F} 1 . \operatorname{Ei}(\mathrm{t})-\mathrm{F} 2 . \text { Eo }(\mathrm{t})
$$

avec Ei $(t)$ excès isotopique du flux entrant au temps $t$ et Eo (t) excès isotopique du flux sortant au temps t.

L'intégration donne :

$$
\frac{\mathrm{q} 1-\mathrm{q} 2}{\mathrm{t} 1-\mathrm{t} 2}=\mathrm{F} 1 \text {.Ëi-F2. Ëo }
$$

avec Ėi excès isotopique moyen du flux entrant et Ēo excès isotopique moyen du flux sortant. Les excès isotopiques moyens peuvent être calculés selon un modèle exponentiel ou linéaire selon la constance ou non des flux. C'est le modèle linéaire que nous utiliserons mais les résultats diffêrent peu de ceux obtenus avec le modèle exponentiel sur cette courte période. Les flux F1 et F2 peuvent être tirés des équations (2) et (6). Il s'agit aussi de flux moyens pour la période d'incubation.

\section{Résultats et discussion}

\subsection{Les bilans}

En ce qui concerne la somme des deux isotopes $\left({ }^{14} \mathrm{~N}\right.$ $+{ }^{15} \mathrm{~N}$ ) l'incubation de 352 heures se traduit par une diminution très nette des compartiments azotés $\left(\mathrm{NH}^{+}\right.$, NO3-, N organique), avec ou sans Tubifex (Tableau 2, Fig. 3). Ce résultat est normal puisque les apports sont interrompus; nous ne sommes plus en état stationnaire. La disparition de matière organique est plus importante dans l'aquarium A et pourrait être attribuée à l'action des oligochètes (sous réserve d'erreur analytique). L'azote ammoniacal total diminue fortement mais pas au profit des nitrates. L'azote ammoniacal adsorbé joue son rôle de tampon et le coefficient de partage $\mathrm{NH}^{+}$adsorbé/NH4+ libre passe de 7,6 à 3,6. L'azote organique dissous augmente légèrement. L'azote nitrique est probablement dénitrifié. 
Tableau 2. Distribution de l'azote dans les différentes classes de composés azotés (quantités en mgN).

Table 2. Nitrogen distribution between the various classes of nitrogen compounds (expressed as Nmg)

\begin{tabular}{llcccccc}
\hline $\begin{array}{l}\text { Résultats } \\
\text { en mgN }\end{array}$ & & $\mathrm{NH}_{4}{ }^{+}$ads. & $\mathrm{NH}_{4}^{+}$libre & $\mathrm{NO}_{3}^{-}$ & $\begin{array}{c}\mathrm{N} \text { org } \\
\text { part. }\end{array}$ & $\begin{array}{l}\mathrm{N} \text { org } \\
\text { diss. }\end{array}$ & Total \\
\hline $\mathrm{T}=0$ & 109 & 43 & 43 & 4156 & 24 & 4376 \\
$\mathrm{~T}=352$ heures & & & & & & & \\
\multicolumn{1}{c}{ AQUARIUM A A EAU } & & 2,5 & 6,4 & & 3,4 & \\
& SED & 68,9 & 19,5 & 0,7 & 3914 & 27,6 & 4033 \\
AQUARIUM S S EAU & & 0,7 & 11,5 & & 2,9 & \\
& SED & 108,8 & 15,8 & 1,2 & 4051 & 28,1 & 4220 \\
\hline
\end{tabular}
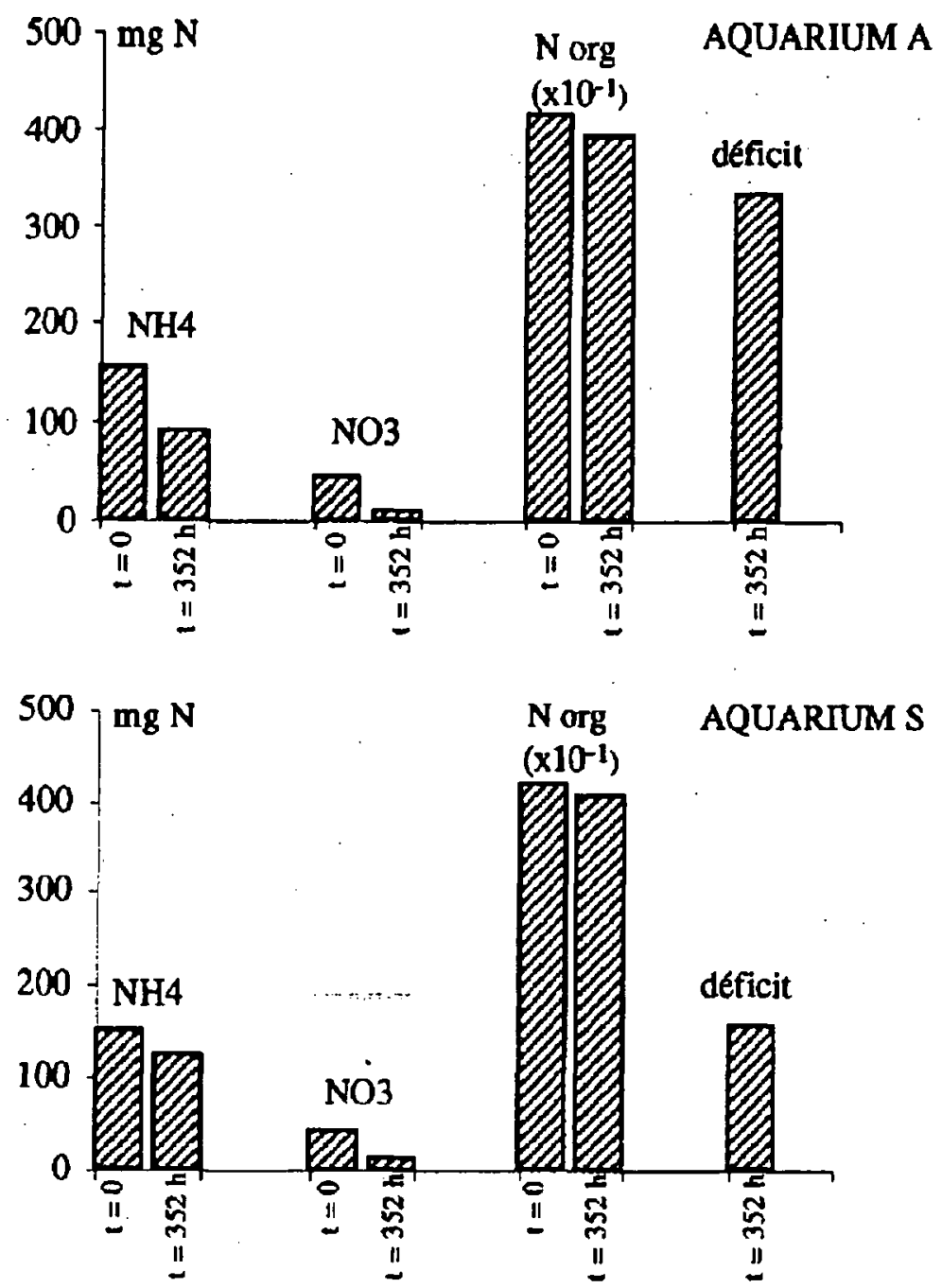

Fig. 3. Répartition de l'azote $(14+15)$ dans les différents compartiments après 352 heures d'incubation.

Fig. 3. $(14+15)$ Nitrogen distribution within the various pools after 352 hours of incubation.

Les bilans d'azote 15 (Tableau 3, Fig. 4) font état de la disparition de $4,1 \mathrm{mg}(19 \%)$ dans l'aquarium $A$ et de $5,2 \mathrm{mg}(25 \%)$ dans l'aquarium S. Contrairement à l'azote léger, les pertes sont plus importantes en l'absence d'oligochètes. Ces pertes ne sont pas retrouvées dans l'azote gazeux ne serait-ce que parce que l'azote NH3 n'a pas été dosé dans l'atmosphère.

Le transfert vers le compartiment nitrate est très faible. Au contraire, le compartiment organique, plus particulièrement dans l'aquarium A, s'enrichit fortement. Les oligochètes favoriseraient donc la réorganisation.

Les pertes et les gains des différents compartiments en azote commun et en azote lourd peuvent être exprimés en flux nets (Tableau 4), la somme algébrique correspondant au déficit. Si l'on calcule l'excès isotopique théorique de l'azote perdu, soit $1,23 \%$ pour l'aquarium $A$ et $3,35 \%$ pour l'aquarium $S$, on voit que la dénitrification n'est pas le seul mécanisme d'émission, étant donné le faible marquage des nitrates. La différence entre les deux aquariums peut s'expliquer par des différences dans le fonctionnement ou par une erreur de dosage, par exemple en sous-estimant le compartiment organique. En effet, une erreur relative (faible) de $1 \%$ correspond à $40 \mathrm{mg}$ d'azote. Une réponse peut être fournie par l'étude des flux bruts à partir de l'analyse du traçage isotopique.

\subsection{Analyse du traçage isotopique}

Contrairement au modèle de Caperon et al. (1979) ou de Blackburn (1979) le modèle utilisé admet une reminéralisation de l'azote 15 organique mais la différence est très faible sur une aussi courte période. Les différents flux ont été calculés soit à partir du compartiment marqué directement $\left(\mathrm{NH}^{+}\right)$soit à partir des compartiments marqués par transferts (matière organique et nitrate). Ils sont représentés sur la figure 5 .

La première constatation est la faiblesse de la nitrification, quel que soit l'aquarium, soit environ $9 \mu$ moles $\mathrm{m}^{-2} \cdot \mathrm{h}^{-1}$. Ce résultat est en accord avec l'impossibilité de mesurer la nitrification sur des carottes de sédi- 
Tableau 3. Excès isotopique et quantité d'azote 15 venue du traceur dans les différents compartiments azotés après 352 heures d'incubation.

Table 3. Isotopic excess and ${ }^{15} \mathrm{~N}$ amounts issued from the tracer measured in the various nitrogenous pools after 352 hours of incubation.

\begin{tabular}{|c|c|c|c|c|c|c|}
\cline { 2 - 7 } \multicolumn{1}{c|}{} & \multicolumn{3}{c|}{ EXCES ISOTOPIQUE (\%) } & \multicolumn{2}{c|}{ QUANTITE D'AZOTE 15(mgN) } \\
\hline AQUARIUM A & AIR & EAU & SED. & AIR & EAU & SED. \\
\hline NH $_{4}$ libre & - & 2,154 & 5,6 & - & 0,037 & 1,092 \\
\hline NH $_{4}$ adsorbé & - & - & 4,691 & - & - & 3,232 \\
\hline $\mathrm{NO}_{3}$ & - & 0,081 & - & - & 0,005 & 0,0005 \\
\hline Norg diss. & - & 0,93 & 0,175 & - & 0,034 & 0,048 \\
\hline Norg part. & - & - & 0,305 & - & - & 12,356 \\
\hline $\mathrm{N}_{2}$ & 0,0087 & - & - & 0,091 & - & - \\
\hline
\end{tabular}

\begin{tabular}{|c|c|c|c|c|c|c|}
\cline { 2 - 7 } \multicolumn{1}{c|}{} & \multicolumn{3}{c|}{ EXCES ISOTOPIQUE (\%) } & \multicolumn{3}{c|}{ QUANTITED'AZOTE 15(mgN) } \\
\hline AQUARIUM S & AIR & EAU & SED. & AIR & EAU & SED. \\
\hline $\mathrm{NH}_{4}$ libre & - & 2,154 & 3,602 & - & 0,015 & 0,569 \\
\hline $\mathrm{NH}_{4}$ adsorbe & - & - & 5,145 & - & - & 5,598 \\
\hline $\mathrm{NO}_{3}$ & - & 0,081 & - & - & 0,009 & 0,0010 \\
\hline Norg diss. & - & 0,764 & 0,132 & - & 0,022 & 0,038 \\
\hline Norg part. & - & - & 0,22 & - & - & 8,98 \\
\hline $\mathrm{N}_{2}$ & 0,0053 & - & - & 0,055 & - & - \\
\hline
\end{tabular}

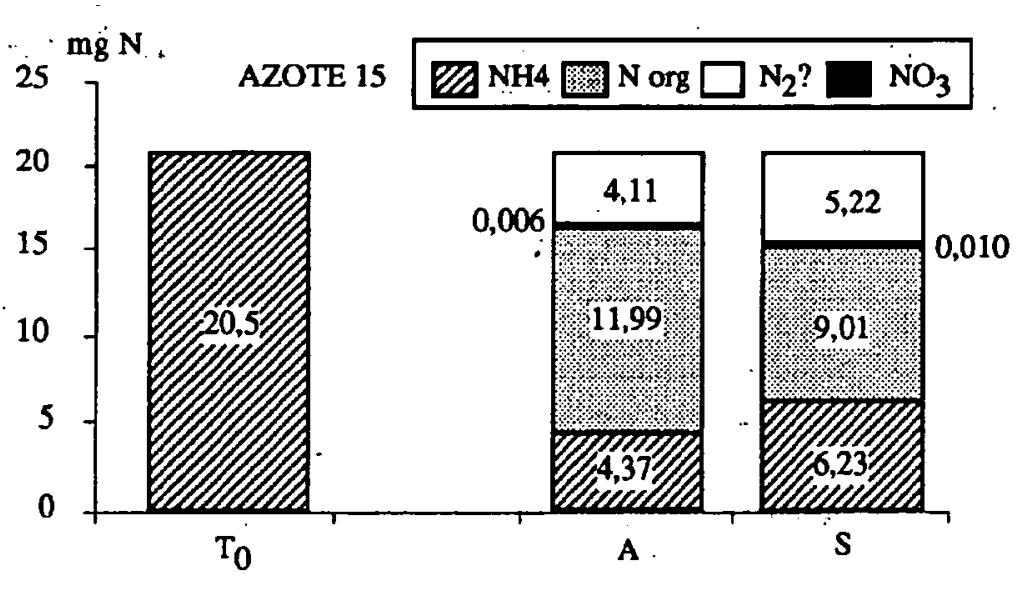

Fig. 4. Répartition de l'azote 15 dans les différents compartiments après 352 heures d'incubation.

Fig. 4. 15 Nitrogen distribution within the various pools after 352 hours of incubation. ments intactes, par différence de production de $\mathrm{NH}_{4}{ }^{+}$ en présence d'acétylène ou d'allylthiourée selon la méthode de Hall (1984). De même, par la fixation de ${ }^{14} \mathrm{CO}_{2}$, selon la méthode de Billen (1976). Boström (1981) observe que la nitrification est très sensible à la turbulence et disparaitt en phase de repos. Cependant, dans son expérience, elle n'est pas négligeable avec une colonne d'eau agitée, 'comme c'est le cas dans notre expérience. Il note l'impossibilité de réalịser en laboratoire les conditions de turbulence aléatoires du lac. Ce phénomène diffère de l'érosion en rivière qui supprime les biofilms actifs (Williamson \& Cooke 1985). La faiblesse de la nitrification s'accompagne d'une perte d'ammoniaque importante. Ce flux est calculé en soustrayant du flux de sortie du compartiment ammoniacal la réorganisation estimée à partir du com- 
Tableau 4. Production nette (+) et disparition nette (-) de l'azote dans les différents compartiments chimiques pendant les 352 heures d'incubation.

Table 4. Net production ( + ) and net loss (-) of nitrogen within the various chimical pools during 352 hours of incubation.

\begin{tabular}{|c|c|c|c|c|}
\hline & $\mathrm{NH}_{4}^{+}$ & $\mathrm{NO}_{3}^{-}$ & $\mathrm{N}$ org & Total \\
\hline \multicolumn{5}{|l|}{ Aquarium A } \\
\hline $\mathrm{N}(14+15)(\mathrm{mg})$ & -63 & -35 & -235 & -333 \\
\hline Flux net (mg. $\left.h^{-1}\right)$ & $-0,179$ & $-0,099$ & $-0,667$ & $-0,945$ \\
\hline $15 \mathrm{~N}(\mathrm{mg})$ & $-16,105$ & $+0,006$ & $+11,986$ & $-4,113$ \\
\hline Flux net $\left(\mathrm{mg} \cdot \mathrm{h}^{-1}\right)$ & $-0,045$ & & $+0,0340$ & $-0,011$ \\
\hline \multicolumn{5}{|l|}{ Aquarium $\mathbf{S}$} \\
\hline $\mathrm{N}(14+15)(\mathrm{mg})$ & -27 & -30 & -99 & -156 \\
\hline Flux net $\left(m g \cdot h^{-1}\right)$ & $-0,076$ & $-0,087$ & $-0,281$ & $-0,444$ \\
\hline $15 \mathrm{~N}(\mathrm{mg})$ & $-14,249$ & $+0,010$ & $+9,014$ & $-5,225$ \\
\hline Flux net $\left(\mathrm{mg} \cdot \mathrm{h}^{-1}\right)$ & $-0,040$ & - & $+0,026$ & $-0,014$ \\
\hline
\end{tabular}

partiment azote organique (la nitrification est négligeable). Cette émission de $\mathrm{NH}^{3}$ correspond aux pertes nettes du compartiment, sans doute favorisées par le «stripping» lié au bullage, la phase gazeuse n'étant pas étanche. Ces pertes ammoniacales expliquent le fort marquage théorique du déficit de bilan global. La dénitrification est environ 100 fois plus importante que la nitrification, quel que soit l'aquarium, et concerne donc des nitrates allochtones. Les flux d'azote calculés, en considérant la réduction assimilative négligeable (Binnerup et al. 1992), soit $1,25 \mathrm{mg} \mathrm{m}^{-2} \mathrm{~h}^{-1}$ dans l'aquarium A et $1,09 \mathrm{mg} \cdot \mathrm{m}^{-2} \mathrm{~h}^{-1}$ dans l'aquarium $\mathrm{S}$ sont un peu inférieurs à ceux mesurés « in situ «, à la même époque, par la méthode à l'acétylène $(1,83 \pm 0,5$ $\left.\mathrm{mg} \cdot \mathrm{m}^{-2} \mathrm{~h}^{-1}\right)$. Il est vrai qu'en l'absence de nitrification, la méthode à l'acétylène sous-estime moins la dénitrification.

Les calculs de la minéralisation et de la réorganisation peuvent être effectués à partir du compartiment organique. Si l'on ne tient pas compte de la variation de taille du compartiment (Laws 1984), les deux flux sont forcément égaux et surtout peu différents de la minéralisation calculée à partir du compartiment ammoniacal dont c'est le flux d'entrée. Par contre, si l'on tient compte de la diminution de ce compartiment organique, le flux de minéralisation est doublé par rapport à celui calculé à partir de la dilution de l'ammonium marqué. Il apparaît donc que le surplus de minéralisation est un artefact lié à une sous-estimation, même faible, de l'azote organique, probablement liée à l'hétérogénéité du compartiment échantillonné.
L'influence de la faune d'oligochètes apparaît faible comparée aux résultats de la littérature qui montrent toujours une augmentation importante de la plupart des flux d'éléments (Pelegri \& Blackburn 1995, Matisoff et al. 1985). Dans notre expérience, la minéralisation et la réorganisation sont nettement accélérées en présence de vers ainsi qu'à un dégré moindre, la dénitrification. Il est possible que leur influence dans l'agitation de l'eau soit masquée par celle du bullage. D' autre part, les populations de vers sont inférieures à celles utilisées par Pelegri \& Blackburn (1995).

\section{Conclusions}

Du point de vue méthodologique, il est évident que le microcosme ne permet pas de reproduire la variabilité aléatoire des conditions d'agitation des lacs peu profonds, soumis à l'influence de tous les vents. Le système d'agitation utilisé (bullage) a peut-être introduit un artefact en favorisant l'émission d'ammoniac (stripping), les aquariums n'étant pas totalement étanches.

Les erreurs analytiques sur l'azote particulaire, probablement liées à l'échantillonnage d'un grand compartiment hétérogène, se traduisent par des modifications considérables des flux de minéralisation. Par contre, la modification du flux d'entrée (réorganisation) est moins importante.

Sur de courtes périodes, le modèle d'évolution du traceur dans les compartiments est sans importance. 


\section{Incubation avec Oligochètes}

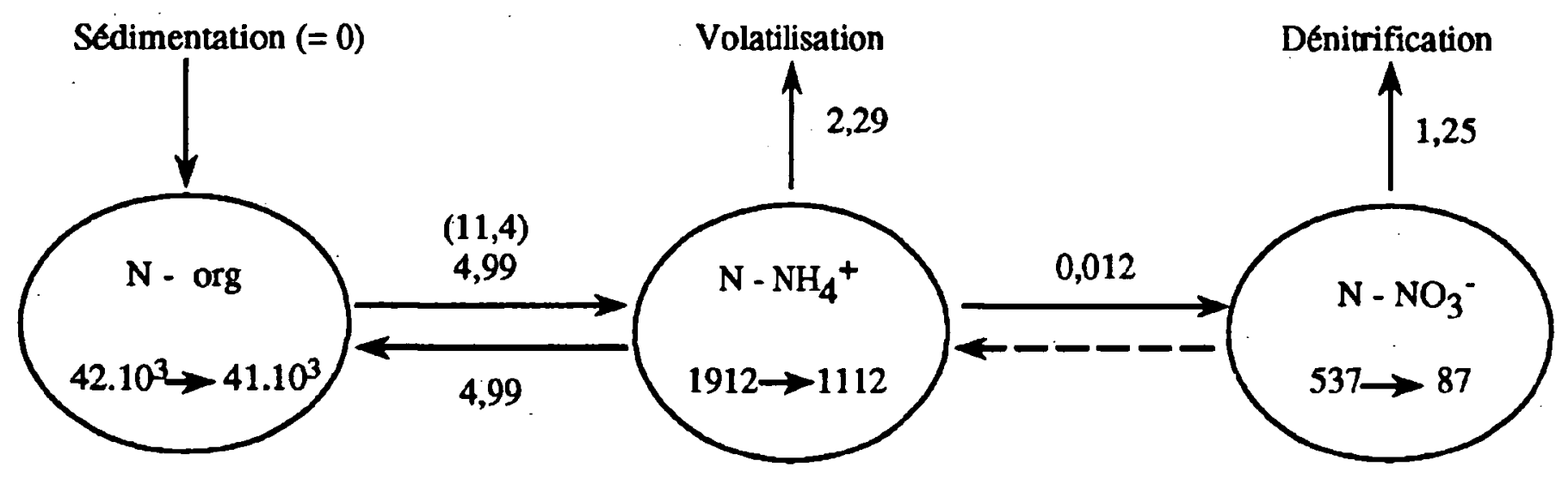

\section{Incubation sans Oligochètes}

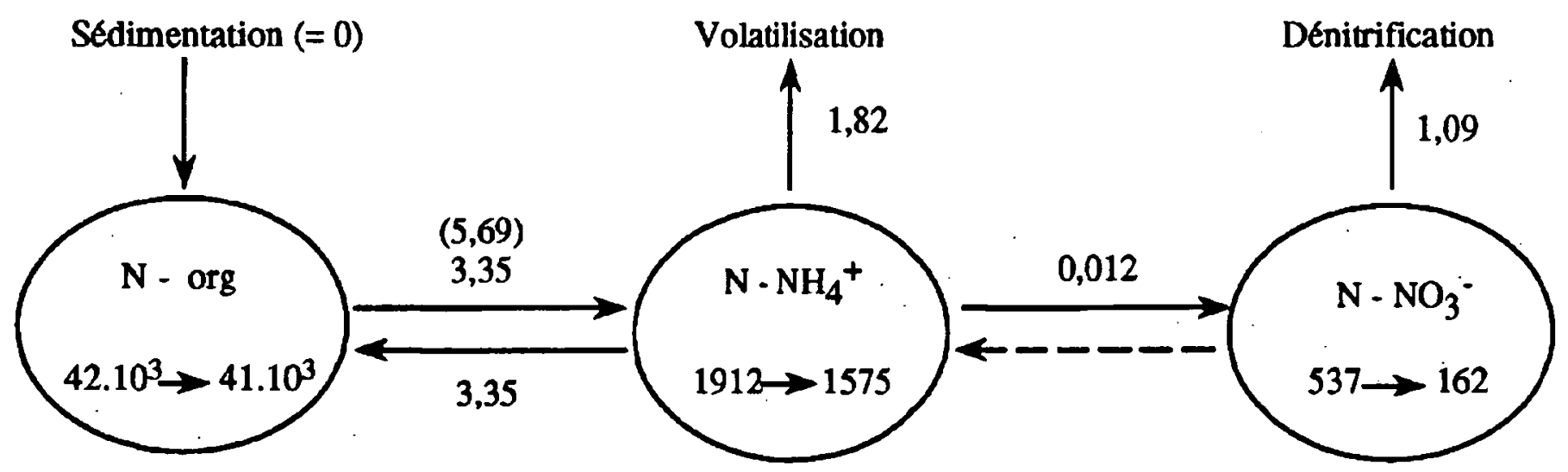

Fig. 5. Compartiments azotés $\left(\mathrm{mg} \cdot \mathrm{m}^{-2}\right)$ et flux $\left(\mathrm{mg} \cdot \mathrm{m}^{-2} \cdot \mathrm{h}-1\right)$ entre les différents compartiments dans les microcosmes de sédiments étudiés. Entre parenthèses, le flux de minéralisation tenant compte de la diminution de l'azote organique.

Fig. 5. Nitrogen pools (mg. $\left.\mathrm{m}^{-2}\right)$ and fluxes $\left(\mathrm{mg} \cdot \mathrm{m}^{-2} \cdot \mathrm{h}^{-1}\right)$ between the pools of the sediment microcosms studied. In parenthesis, the mineralization flux accounting for the organic nitrogen diminution.

Du point de vue des flux, la faiblesse de la nitrificátion confirme les résultats déjà obtenus par d'autres techniques. Peut-être se produit-elle pendant les phases de resuspension? Elle est probablement secondaire vis-à-vis des apports de la nappe qui alimentent la dénitrification. Les résultats obtenus pour ce processus sont proches de ceux obtenus in situ par la méthode à l'acétylène. Dans les lacs de gravière colmatés par les fines de lavage, le flux de nitrates de la nappe peutêtre insuffisant en été et la dénitrification disparaît avec les nitrates de la colonne d'eau. Cependant, la croissance des algues n'est pas interrompue, soit parce que le flux de la nappe leur suffit (elles sont mieux placées que les bactéries des sédiments pour l'utiliser), soit parce que le flux d'ammonium issu des sédiments assure cette croissance.

L'influence des Oligochètes semble se manifester très positivement au niveau du couple minéralisation réorganisation et plus faiblement, au niveau de la dénitrification.

Cependant, dans nos conditions expérimentales, les effets sont moins spectaculaires que ceux décrits dans la littérature: 


\section{Travaux cités}

Bergé P. 1983. - Détection de l'origine des pollutions azotées d'un aquifere alluvial de la vallée de la Garonne par traçage isotopique. Thèse $3^{\mathrm{e}}$ cycle, Toulouse : $221 \mathrm{p}$.

Billen G. 1976. - Evaluation of nitrifying activity in sediments by dark ${ }^{14} \mathrm{C}$ bicarbonate incorporation. Water Res., $10: 51-57$.

Blackburn T.H. 1979. - Method for measuring rates of $\mathrm{NH}^{+}$turnover in anoxic marine sediments, using a ${ }^{15} \mathrm{~N}-\mathrm{NH} 4^{+}$dilution technique. Appl. Environ. Microbiol., 37 : 760-765.

Boström B. 1981. - Factors controlling the seasonal variation of nitrate in lake Erken. Int. Revue ges. Hydrobiol., 66 (6), 821-836.

Binnerup S.J., Jensen K., Revsbech N.P., Jensen M.H. \& Sorensen J. 1992. - Denitrification, dissimilatory reduction of nitrate to ammonium and nitrification in a bioturbated estuarine sediment as measured with ${ }^{15} \mathrm{~N}$ and microsensor techniques. Appl. Environ. Microbiol., 58 : 303-313.

Blackburn T.H. 1979. - Method for measuring rates of $\mathrm{NH}_{4}{ }^{+}$turnover in anoxic marine sediments, using a $15 \mathrm{~N}^{-\mathrm{NH}_{4}+}$ dilution technique. Appl. Environ. Microbiol., 37 : 760-765.

Bremmer J.M. 1965. - Total nitrogen, in Methods of soil analysis, Black C.A. et al. Ed., American Society of Agronomy, Inc. Publisher, Madison, Wisconsin USA, ch. 83 : 1149-1286.

Caperon J., Schell D., Hirota J. \& Laws E. 1979. - Ammonium excretion rates in Kaneohe Bay, Hawai, measured by a ${ }^{15} \mathrm{~N}$ isotope dilution technique. Mar. Biol, , $54: 33-40$.

Chen R.L., Keeney D.R., Graetz D.A. \& Holding A.J. 1972. -Denitrification and nitrate reduction in Wisconsin lake sediments. $J$. Environ. Qual., $1: 158-162$.

Dugdale R.C. \& Goering J.J. 1967. - Uptake of new and regenerated forms of nitrogen in primary productivity. Limnol. Oceanogr., $12: 196-206$.

Fardeau J.C. \& Jappe J. 1982. — Intérêt des cinétiques d'échanges isotopiques pour la caractérisation du phosphore assimilable des sols. Sciences du Sol, 2 : 113-124.

Gardner W.S., Nalepa T.F., Slavens D.R. \& Laird G. 1983. — Patterns and rates of nitrogen release by benthic chironomids and oligochaeta. Can. J. Fish. Aquat. Sci., $40:$ 259-266.

Glibert P.M., Lipschultz F., McCarthy J.J. \& Altabet M.A. 1982. Isotope dilution models of uptake and remineralization of ammonium by marine phytoplancton. Limnol. Oceanogr., 27 : 639-650.

Goering J.J \& Dugdale R.C. 1966. - Denitrification rates in a island bay in the equatorial Pacific Ocean. Science, $154: 505$.

Goering J.J \& Pamatmat M.M. 1971. - Denitrification in sediments of the sea off Peru. Invest. Pesq., $35: 233-242$.

Guérin C. \& Labroue L. 1991. - Etude expérimentale de l'influence de Tubifex tubifex Müll. (Tubificidae, Oligochaeta) sur le relargage du phosphore par le sédiment. Annls. Limnol., 27 (3) : 253 265 .
Guiraud G. 1984. - Contribution du marquage isotopique à l'évaluation des transferts d'azote entre les compartiments organiques et minéraux dans les systèmes sol-plante. Thèse, Paris VI : $335 \mathrm{p}$.

Keeney D.R., Chen R.L. \& Graetz D.A. 1971. - Importance of denitrification and nitrate reduction in sediments to the nitrogen budgets of lakes. Nature (London), $233: 66-67$

Kirkham D. \& Bartholomew W.V. 1954. - Equations for following nutrient transformations in soil, utilizing tracer data. Soil Sci. Am. Proc., $18: 33-34$

Kirkham D. \& Bartholomew W.V. 1955. -Equations for following nutrient transformations in soil, utilizing tracer data. II. Soil Sci. Soc. Am. Proc., 19 : 189-192.

Koike I. \& Hattori H. 1978. - Simultaneous determination of nitrification and nitrate reduction in coastal sediments by ${ }^{15} \mathrm{~N}$ dilution technique. Appl. Environ. Microbiol., 35 : 853-857.

Labroue L., Tourenq J.N., Mieussens C., Robert J. \& Donville B. 1988. - Rôle des lacs de gravière dans la diminution des teneurs en nitrates des aquiferes alluviaux de la vallée de la Garonne. Essai de quantification. Annls Limnol., 24 (1) : 31-38.

Labroue L., Vidal W., Tosca C. \& Bergé Ph. 1985. - Transferts d'azote minéral dans les sols froids : essai de traçage isotopique in situ : Soil Biol. Biochem., 17, (5), 683-689.

Laws E. 1984. - Isotope dilution models and the mystery of vanishing ${ }^{15}$ N. Limnol. Oceanogr., 29 (2), 379-386.

Madsen P.P. 1979. - Seasonal variation of denitrification rate in sediment determined by use of ${ }^{15} \mathrm{~N}$. Water Res., 13: 461-465.

Mariotti A., Letolle R. \& Guillemont J. 1978. - Analyse isotopique de l'azote au niveau des abondances naturelles. Analusis., 6 (10) 421-425.

Matisoff G., Fisher J.B. \& Matis S. 1985. - Effects of benthic macroinvertebrates on the exchange of solutes between sediments and freshwater. Hydrobiologie, $122: 19-33$.

Oren A. \& Blackburn T.H. 1979. - Estimation of sediment denitrification rates at in situ nitrate concentration. Appl. Environ. Microbiol., 37 : 174-176.

Pelegri S.P. \& Blackburn T.H. 1995. - Effects of Tubifex tubifex (Oligochaeta : Tubificidae) on $\mathrm{N}$-mineralization in freshwater sediments, measured with ${ }^{15} \mathrm{~N}$ isotopes. Aquat. microb. Ecol., 9 : 289-294.

Sørensen J. 1978. - Capacity for denitrification and reduction of nitrate to ammonia in a coastal marine sediment. Appl. Environ. Microbiol., $35: 301-305$.

Sumi T. \& Koike I. 1990. -Estimation of ammonification and ammonium assimilation in surficial coastal and estuarine sediments Limnol. Oceanogr., 35 (2), 270-286.

Tiren T., Thorin J. \& Nömmik H. 1976. - Denitrification measurements in lakes. Acta Agric. Scandi., 26: 175-184.

Williamson R.B. \& Cooke J.G. 1985. - The effect of storms on nitrification rates in a small stream. Water Res., 19 (4), 435-446. 